\title{
OPEN ASPHERICAL MANIFOLDS NOT COVERED BY THE EUCLIDEAN SPACE
}

\author{
IGOR BELEGRADEK
}

\begin{abstract}
We show that any open aspherical manifold of dimension $n \geq 4$ is tangentially homotopy equivalent to an $n$-manifold whose universal cover is not homeomorphic to $\mathbb{R}^{n}$.
\end{abstract}

\section{INTRODUCTION}

Davis famously constructed, for every $n \geq 4$, a closed aspherical $n$-manifold whose universal cover is not homeomorphic to $\mathbb{R}^{n}$ Dav83. We prove:

Theorem 1.1. If the universal cover of an open smooth manifold $V$ is diffeomorphic to $\mathbb{R}^{n}$ with $n \geq 4$, then the tangential homotopy type of $V$ contains a continuum of open smooth $n$-manifolds whose universal covers are not homeomorphic to $\mathbb{R}^{n}$.

Our proof also works in PL and TOP categories. Recall that a homotopy equivalence $f: V \rightarrow W$ of $n$-manifolds is tangential if $f^{\#} T W$ and $T V$ are stably isomorphic, where $T X$ denotes the tangent bundle if $X$ is a smooth manifold and the tangent microbundle in the PL or TOP cases. By [Sie69, Theorem 2.3] this is equivalent to requiring that $f \times \mathbf{i d}\left(\mathbb{R}^{s}\right)$ is homotopic to a CAT-homeomorphism for some $s$ (where CAT equals DIFF, PL or TOP).

In the simply-connected case Theorem [1.1 is due to Curtis-Kwun [CK65] for $n \geq 5$ and to Glasner [Gla67] for $n=4$. The proof combines three ingredients:

(1) A result of Curtis-Kwun CK65 that for a boundary connected sum $S$ of a countable family of compact $n$-manifolds, the homeomorphism type of $\operatorname{Int}(S)$ determines the isomorphism class of $\pi_{1}(\partial S)$.

(2) A recent result of Calcut-King-Siebenmann CKS12 that any countable collection of CAT properly embedded $\mathbb{R}^{n-1}$ 's in $\mathbb{R}^{n}$ is CAT unknotted, which generalizes classical results of Cantrell and Stallings.

2000 Mathematics Subject classification. Primary 57N15. Keywords: aspherical manifold, homology sphere, hyperplane unknotting. The author is grateful for NSF support (DMS1105045). 
(3) The existence of infinitely many smooth compact contractible $n$-manifolds whose boundary homology spheres have freely indecomposable fundamental groups. (Such examples can be found in CK65, Gla67, and more examples are now known, see e.g. Casson-Harer CH81 for aspherical homology 3-spheres that bound smooth contractible 4-manifolds, while Kervaire Ker69 showed that the fundamental group of any homology 3 -sphere appears as the fundamental group of the boundary of a smooth contractible $n$-manifolds for any $n \geq 5)$.

Proof. Since $V$ is open, it contains a CAT properly embedded ray whose CAT regular neighborhood is an embedded closed halfspace, see e.g. CKS12, Section $3]$. Hence $V$ is CAT isomorphic to the interior of a noncompact manifold $N$ whose boundary is an open disk. By the strong version of the Cantrell-Stalling hyperplane unknotting theorem proved in CKS12, Corollary 9.3], the universal cover of $N$ can be compactified to $D^{n}$, the $n$-disk, in which the preimage of $\partial N$ becomes the union of a countable collection of round open disks with pairwise disjoint closures.

Let $\left\{C_{i}\right\}_{i \in \mathbb{N}}$ be an infinite sequence of compact contractible $n$-manifolds, such that $\pi_{1}\left(\partial C_{i}\right)$ are pairwise non-isomorphic and freely indecomposable. Given a subset $\alpha \subseteq \mathbb{N}$, let $C_{\alpha}$ be a boundary connected sums of $C_{i}$ 's with indices in $\alpha$. (For our purposes the choices involved in defining boundary connected sums will always be irrelevant). Fix a closed $(n-1)$-disk $\Delta \subset \partial C_{\alpha}$, and let $N_{\alpha}$ be a boundary connected sum of $N$ and $C_{\alpha}$ obtained by identifying $\Delta$ with a closed disk in $\partial N$. A deformation retraction $C_{\alpha} \rightarrow \Delta$ extends to a deformation retraction of $N_{\alpha} \rightarrow N$, so $V_{\alpha}:=\operatorname{Int}\left(N_{\alpha}\right)$ is tangentially homotopy equivalent to $V$.

If $Q_{\alpha}$ denotes a boundary connected sum of countably many copies of $C_{\alpha}$, then the interior of the universal cover of $N_{\alpha}$ is homeomorphic to the interior of a boundary connected sum of $D^{n}$ and $Q_{\alpha}$, which is homeomorphic to $\operatorname{Int}\left(Q_{\alpha}\right)$. By [CK65, Theorem 4.1] if $Q_{\alpha}, Q_{\beta}$ have homeomorphic interiors, then $\partial Q_{\alpha}$, $\partial Q_{\beta}$ have isomorphic fundamental groups. Now $\pi_{1}\left(\partial Q_{\alpha}\right)$ is a free product in which each factor $\pi_{1}\left(\partial C_{i_{k}}\right), i_{k} \in \alpha$ appears countably many times. Each $\pi_{1}\left(\partial C_{i_{k}}\right)$ is freely indecomposable, so $\alpha=\beta$ by Grushko's theorem. Thus the universal covers of $\operatorname{Int}\left(N_{\alpha}\right)$ lie in a continuum of homeomorphism types.

Remark 1.2. The proof of [CK65, Theorem 4.1] is quite technical, which may be due to the fact that the tools of Siebenmann's thesis were not yet available at the time, so we summarize it in a modern language: Given $\alpha=\left\{i_{1}, \ldots, i_{k}, \ldots\right\}$ it is easy to construct a cofinal family $\left\{U_{k}\right\}_{k \geq 1}$ of neighborhoods of infinity in $Q_{\alpha}$ such that in the corresponding inverse sequence of fundamental groups, the group $\left\{\pi_{1}\left(U_{k}\right)\right\}$ is the free product of $\pi_{1}\left(C_{i_{1}}\right) * \cdots * \pi_{1}\left(C_{i_{k}}\right)$, and the map $\pi_{1}\left(U_{k}\right) \longleftarrow \pi_{1}\left(U_{k+1}\right)$ is a retraction onto the first $k$ factors, and in particular, is 
surjective. Hence the inverse sequence of groups is Mittag-Leffler, and therefore its pro-equivalence class depends only on the homeomorphism type of $Q_{\alpha}$. Now if $Q_{\alpha}, Q_{\beta}$ have homeomorphic interiors, then a simple diagram chase in the commutative diagram from the definition of pro-equivalence shows that each free factor of $\pi_{1}\left(Q_{\alpha}\right)$ occurs as a free factor of $\pi_{1}\left(Q_{\beta}\right)$, so $\alpha=\beta$.

Remark 1.3. Theorem 1.1 should hold for $n=3$, but our proof fails. One could try substituting the boundary connected sum of $N$ and $Q_{\alpha}$ by the end sum of $V$ with a suitable Whitehead manifold, but the multiple hyperplane unknotting is no longer true, due to existence of an exotic $[0,1] \times \mathbb{R}^{2}[$ ST89]. This makes analyzing the fundamental group of infinity more delicate. For the same reason we do not attempt to prove Theorem 1.1 for $V$ whose universal cover is not $\mathbb{R}^{n}$.

Remark 1.4. By the Cartan-Hadamard theorem any (complete Riemannian) $n$-manifold of nonpositive curvature is covered by $\mathbb{R}^{n}$. So given an open $n$ manifold of nonpositive curvature with $n \geq 4$, Theorem 1.1 yields a continuum of $n$-manifolds in the same tangential homotopy type that admit no metric of nonpositive curvature. In [Bel] the author studied obstructions to nonpositive curvature on open manifolds that go beyond the Cartan-Hadamard theorem.

\section{REFERENCES}

[Bel] I. Belegradek, Obstructions to nonpositive curvature for open manifolds, arXiv:1208.5220v1.

[CH81] A. J. Casson and J. L. Harer, Some homology lens spaces which bound rational homology balls, Pacific J. Math. 96 (1981), no. 1, 23-36.

[CK65] M. L. Curtis and K. W. Kwun, Infinite sums of manifolds, Topology 3 (1965), 31-42.

[CKS12] J. S. Calcut, H. C. King, and L. C. Siebenmann, Connected sum at infinity and Cantrell-Stallings hyperplane unknotting, Rocky Mountain J. Math., 42 (2012), no. 6, 1803-1862.

[Dav83] M. W. Davis, Groups generated by reflections and aspherical manifolds not covered by Euclidean space, Ann. of Math. (2) 117 (1983), no. 2, 293-324.

[Gla67] L. C. Glaser, Uncountably many contractible open 4-manifolds, Topology 6 (1967), $37-42$.

[Ker69] M. A. Kervaire, Smooth homology spheres and their fundamental groups, Trans. Amer. Math. Soc. 144 (1969), 67-72.

[ST89] P. Scott and T. Tucker, Some examples of exotic noncompact 3-manifolds, Quart. J. Math. Oxford Ser. (2) 40 (1989), no. 160, 481-499.

[Sie69] L. C. Siebenmann, On detecting open collars, Trans. Amer. Math. Soc., 142, (1969), 201-227.

Igor Belegradek, School of Mathematics, Georgia Institute of Technology, AtLANTA, GA 30332-0160

E-mail address: ib@math.gatech.edu 